\title{
Rapport des réunions du EAP
}

Vienne, 21-24/2/2019

Peter Schulthess

Chaque année, les délégués de l'Association européenne pour la psychothérapie (European Association for Psychotherapy - EAP) se réunissent pour les réunions de février à Vienne dans les locaux de l'Université privée Sigmund Freud (SFU). Le signataire et le président représentent tous conjointement l'ASP auprès du Conseil général (Conseil élargi) et au Comité national des organisations faîtières (NUOC).

Répartis sur cinq jours (deux comités se réunissent juste une journée avant les réunions officielles), un total de 32 réunions des différents comités et groupes de travail ont eu lieu cette fois. Pour ceux qui participent à plusieurs comités ou groupes de travail thématiques, un programme considérable!

La réunion du Conseil d'administration a débuté par une minute de silence pour le professeur Rodolfo de Bernart. Il est décédé le 19 février 2019. Le professeur de Bernart a été directeur de l'Institut de thérapie familiale à Florence et a enseigné la thérapie familiale dans le domaine de la psychiatrie à l'Université de la ville. Il a été président sortant de la société européenne de thérapie familiale (Europäischen Gesellschaft für Familientherapie - EFTA) et de l'EAP.

Le groupe marketing a présenté une apparition sur Facebook au Conseil d'administration. Elle a plaidé pour une présence sur ce canal de médias sociaux pour obtenir plus de publicité pour l'EAP. Cela a donné lieu à des discussions et des questions fondamentales sur la façon de contrôler la dynamique dans les graphiques. Une page interactive sur Facebook a besoin d'un soutien compétent et doit également être en mesure de répondre rapidement aux questions techniques, telles que celles qui sont souvent adressées au secrétariat. Le projet sera développé et présenté à nouveau à la prochaine réunion. Parallèlement à l'apparition sur Facebook, le site sera révisé, également sous la direction du groupe marketing.

Le Conseil a également été informé des problèmes juridiques liés aux psychothérapies basées sur Internet. Il convient de noter que seules des connexions Internet sécurisées doivent être utilisées, sinon le secret professionnel ne peut pas être maintenu. Skype est interdit car ce système ne garantit pas la confidentialité. Zoom ou VSee sont recommandées. WhatsApp est également une option à prendre en considération, car ce système utilise un cryptage de périphérique à périphérique depuis un certain temps. En tant quadresse Internet pour le trafic de courrier électronique, les membres ASP peuvent configurer une adresse HIN qui a longtemps été utilisée comme connexion de messagerie sécurisée pour la communication entre médecins.

Dans le cas de contacts internationaux, le droit de l'État dans lequel vit le client est applicable. Les psychothérapeutes doivent donc avoir une formation équivalente à celle des thérapeutes dans ce pays. Presque personne n'en est conscient. Le thème a été approfondi dimanche lors d'une réunion pour les instituts de formation EAP.

Il existe désormais des cours de formation (sur Internet, bien sûr) sur la façon de gérer en toute sécurité les thérapies en ligne, et de transmettre ce qui doit être considéré. Les diplômés de ces cours sont agréés en tant que spécialistes en thérapie par Internet.

Les activités du lobbyiste de l'EAP en coopération avec le groupe du lobby ont également été rapportées. La première étape a été « l'European Act of Psychotherapy » ( l'acte européen de la psychothérapie) qui confère à l'EAP, le statut d’organisation européenne compétente responsable de la psychothérapie au Parlement européen. D’autres étapes sont en préparation.

En Espagne, la législation sur la psychothérapie est en suspens. Le conflit habituel entre les associations de psychologie et les associations de psychothérapeutiques a pris fin ; qui pourrait être le représentant légitime de la psychothérapie et donc un partenaire de négociation avec le ministère de la Santé : la Fédération européenne des associations de psychologie (EFPA) ou l'EAP ou leurs associations nationales. Le Seplis, lorganisation faittière européenne pour les professions libérales, dans laquelle l'EFPA et l'EAP sont membres, tente de bâtir un pont. Une publication de l'EFPA énumère les compétences professionnelles des psychologues et celles des psychothérapeutes. L'EFPA documente également qu'il s'agit de deux professions différentes. Il sera intéressant de voir comment cela se poursuivra. Dans le cadre d'une conférence distincte pour les instituts de formation EAP, il a été une nouvelle fois énoncé comment l'academisation de la psychothérapie progresse et est imparable. Dans divers pays européens, des cours universitaires en psychothérapie permettent d'acquérir des qualifications professionnelles. L'Allemagne vient d'adopter une révision correspondante de

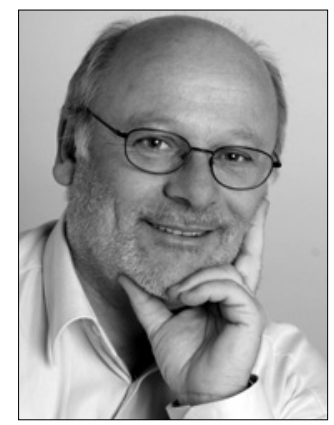




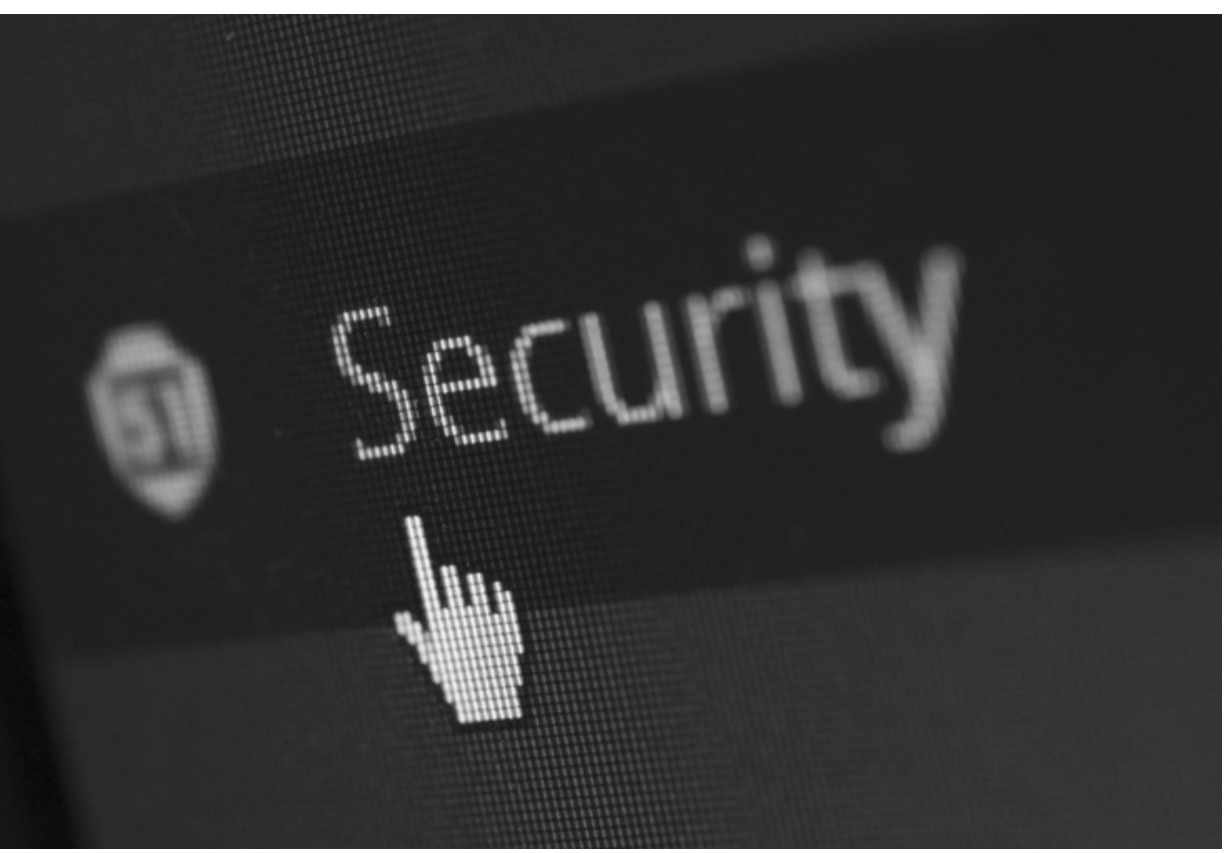

la loi. Il est donc essentiel que les programmes de formation soient fondés sur le cadre européen de qualification (European Qualification Framework - EQF) niveau 7 (maîtrise universitaire). Cela exige que les modules pour la recherche en psychothérapie soient également intégrés dans les programmes de formation et qu'une thèse soit au niveau de celle d'une mâ̂trise. Certains instituts ont déjà réalisé ce développement, d'autres ne l'ont pas encore fait. Le sujet devrait être approfondi et mis en œuvre lors de réunions ultérieures. Le Comité des sciences et de la recherche (SARC) coordonne les échanges entre les instituts.

Le SARC a également indiqué qu'il avait été sollicité par l'organisation faîtière espagnole (l'équivalent de l'ASP) pour apporter un soutien au ministère de la santé dans le cadre de la prochaine réglementation juridique de la psychothérapie. $\mathrm{Si}$ alors des avis sur la question estiment néanmoins que la psychothérapie est scientifiquement non fondée. Les associations ont été obligées de montrer des preuves empiriques de la psychothérapie. Grâce à son réseau international regroupant les principaux chercheurs en psychothérapie du monde entier, le SARC a réussi à fournir de nombreux documents pertinents sur l'état de la recherche en psychothérapie, aidant ainsi l'organisation faîtière espagnole. Cette abondance de références bibliographiques doit désormais être systématisée et préparée de manière à être disponible sur le site Web de l'EAP, à la fois pour le bénéfice des organisations affiliées et en tant que source des ministères de la Santé. Les organisations faîtières européennes responsables de diverses orientations psychothérapeutiques, ont été invitées à transmettre au SARC des examens portant sur des méthodes connues afin que ces études puissent également être incluses.

Lors de l'assemblée générale annuelle, il a été également nécessaire de s'occuper des affaires statutaires. Charles Cassar a été confirmé comme président. Particia Hunt de la Grande-Bretagne, a été élue nouvelle vice-présidente. Il prendra le relais du bureau dans deux ans. Depuis la démission de l'ancien président Philip Vranken, le poste de deuxième vice-président a été vacant. Celia Scanlan était prête à apporter sa contribution extraordinaire pour deux ans de plus. Dans le cas contraire, les fonctionnaires ont été confirmés dans leurs bureaux. Le débat sur le budget a suscité beaucoup d'attention, selon lequel les postes du SARC et du International Journal for Psychotherapy (IJP) devraient être réduits à deux postes, étonnamment sur recommandation du groupe de travail financier et du comité exécutif. Le budget du SARC a été défendu avec succès, mais le journal a dû accepter une réduction importante. Le montant manquant doit être compensé par la conversion en un journal entièrement numérique.

Certains amendements ou ajustements ont été approuvés sans discussion, en particulier l'augmentation du nombre de membres du Comité exécutif, de sorte que d'autres présidents de comités puissent désormais y siéger. En outre, les instituts de formation ont finalement reçu leur propre chambre.

Peter Schulthess est membre du Conseil d'administration de l'ASP et le représente avec Gabriela Rüttimann au sein de l'EAP. Il est également président du SARC au sein de l'EAP. 